\title{
Quantum algorithms for computational nuclear physics revisited, particular case of second quantized formulation
}

\author{
Jakub Višňák ${ }^{1,2,3^{*}}$, Petr Veselý $^{4}$ \\ ${ }^{1}$ Dep. of Nuclear Chemistry, FNSPE, Czech Technical University, Břehová 7, 11519 Prague 1, Czech Rep. \\ ${ }^{2}$ J. Heyrovský Institute of Physical Chemistry, Dolejškova 2155/3, 18223 Prague 8, Czech Rep. \\ ${ }^{3}$ Dep. of Chem. Phys. and Optics, FMP, Charles University in Prague, 12116 Prague 2, Czech Rep. \\ ${ }^{4}$ Nuclear Physics Institute, Czech Academy of Sciences, 25068 Rež, Czech Rep.
}

\begin{abstract}
No core Full Configurational Interaction (NCFCI) calculations of Nuclear Bonding energy are resource demanding, in particular, computational time scales exponentially with the nucleon number $A$. In contrast to that, usage of quantum computers would allow an efficient (in polynomial time) NCFCI calculation and speed-up for other beyond-Mean-Field (correlation energy including) methods. To initiate feasibility studies of given quantum algorithms, we present an introduction to preliminary classicalcomputer simulation for the case of spherical nuclei (and ${ }^{4} \mathrm{He}$ in particular) within NCFCI with realistic chiral NNLO_opt potential.
\end{abstract}

\section{Motivation}

Due to the exponential scaling of possible configuration number, accurate NCFCI calculations done on classical computers have been limited up to the p-shell nuclei $[1,2,14]$. Quantum computers [3] could change that, allowing heavier nuclei calculations, since they offer exponential speed-up - to demonstrate that, AbramsLloyd algorithm [4-8] adopted to Computational Nuclear Physics realm will be presented followed by first computational steps towards greater feasibility study for the ${ }^{4} \mathrm{He}$ nuclei.

\section{Problem formulation}

\subsection{Configuration interaction}

General nuclear structure hamiltonian in second quantization (considering No-pair approximation and nucleonic structure with at most k-body interactions) has a form

$$
\hat{H}=\sum_{n=1}^{k} \sum_{\mathbf{i}_{n}} \sum_{\mathbf{j}_{n}} V^{(n)}\left(\mathbf{i}_{n}, \mathbf{j}_{n}\right) \hat{a}^{+}\left(\mathbf{i}_{n}\right) \hat{a}\left(\mathbf{j}_{n}\right),
$$

where $\mathbf{j}_{\mathrm{n}}$ stands for $n$-tuple of integer indices $\left(j_{l}, j_{2}, \ldots\right.$, $\left.j_{n}\right), \quad \hat{a}\left(\mathbf{j}_{n}\right)$ for a string of annihilation operators (2) and $\hat{a}^{+}\left(\mathbf{i}_{n}\right)$ for a string of creation operators (3), $V^{(n)}\left(\mathbf{i}_{n}, \mathbf{j}_{n}\right)$ is a complex coefficient (4),

$$
\begin{aligned}
& \hat{a}\left(\mathbf{j}_{n}\right)=\hat{a}\left(j_{1}\right) \hat{a}\left(j_{2}\right) \quad \hat{a}\left(j_{n}\right), \\
& \hat{a}^{+}\left(\mathbf{i}_{n}\right)=\hat{a}^{+}\left(i_{n}\right) \hat{a}^{+}\left(i_{n-1}\right) \quad \hat{a}^{+}\left(i_{1}\right), \\
& V^{(n)}\left(\mathbf{i}_{n}, \mathbf{j}_{n}\right)=\left\langle i_{1}, i_{2}, \quad, i_{n}\left|\hat{V}^{(n)}\right| j_{1}, j_{2}, \quad, j_{n}\right\rangle,
\end{aligned}
$$

the creation/annihilation refers to $w$ complete orthonormal (ON) sets of one-particle functions (defined on spatial and spin-1/2 domains) - for an ordinary nuclei $w=2$, one set corresponding to protons, one to neutrons (e.g. we can reserve negative integers for the former and positive for latter). In the standard formulation, number of creation and annihilation operators for the given type of nucleon must be same in each term in (1). Creation and annihilation operators, as connected to two distinct types of fermions has to obey anticommutation relations (5) and commute with operators for the other nucleonic type

$$
\begin{aligned}
& {\left[\hat{a}^{+}(i), \hat{a}(j)\right]_{\operatorname{sign}(i \cdot j)} \equiv} \\
& \equiv \hat{a}^{+}(i) \hat{a}(j)+\operatorname{sign}(i \cdot j) \hat{a}(j) \hat{a}^{+}(i)=\delta(i, j) \\
& {[\hat{a}(i), \hat{a}(j)]_{\operatorname{sign}(i \cdot j)}=0 .}
\end{aligned}
$$

While particular example tested consists of at most 2-body interactions ( $k=2$, the simplest non-trivial), the quantum algorithm presented can be generalized for any $k$ (and any $w$ ). In (4) the complex coefficient is therefore identified with matrix element of $n$-body force operator in one-particle $\mathrm{ON}$ basis set. Due to the indistinguishability of identical nucleons and hermitian character of hamiltonian, coefficients $V^{(\mathrm{n})}$ must obey relations

$$
V^{(n)}\left(\mathbf{i}_{n}, \mathbf{j}_{n}\right)=V^{(n)}\left(P \mathbf{i}_{n}, P \mathbf{j}_{n}\right)=\left(V^{(n)}\left(\mathbf{j}_{n}, \mathbf{i}_{n}\right)\right)^{*},(7)
$$

where $P$ is an arbitrary permutation of identical nucleon indices. For presented case of $k=2$ and $w=2 \mathrm{ON}$ set of Hartree-Fock (HF, Mean-field, [9-11]) solutions for the ground state of double magic nuclei has been used, starting with ${ }^{4} \mathrm{He}$. The HF equations has following form 


$$
\begin{aligned}
& \hat{F}^{(p)}\left|\psi_{i}^{(p)}\right\rangle=\varepsilon_{i}^{(p)}\left|\psi_{i}^{(p)}\right\rangle, \\
& \hat{F}^{(n)}\left|\psi_{i}^{(n)}\right\rangle=\varepsilon_{i}^{(n)}\left|\psi_{i}^{(n)}\right\rangle,
\end{aligned}
$$

where Fockians ("p" for protons, "n" for neutrons) in arbitrary ON basis sets $\left\{\left|\phi_{\mu}^{(r)}\right\rangle \mid \mu \in I^{(r)}\right\}(r \in\{\mathrm{p}, \mathrm{n}\})$ has the form

$$
\begin{aligned}
F_{\mu \nu}^{(r)} & =\left\langle\phi_{\mu}^{(r)}\left|h^{(r)}\right| \phi_{\nu}^{(r)}\right\rangle+ \\
+ & \sum_{\sigma \in o c c(r)}\left\langle\phi_{\mu}^{(r)} \psi_{\sigma}^{(r)}\left\|V^{(r r)}\right\| \phi_{\nu}^{(r)} \psi_{\sigma}^{(r)}\right\rangle+ \\
+ & \sum_{\sigma \in o c c(s)}\left\langle\phi_{\mu}^{(r)} \psi_{\sigma}^{(s)}\left|V^{(r s)}\right| \phi_{\nu}^{(r)} \psi_{\sigma}^{(s)}\right\rangle,
\end{aligned}
$$

where $V^{(1)}=h^{(r)}$ represents operator of kinetic energy, second line on right side (10) is an antisymmetrized matrix element of proton-proton (for $r=\mathrm{p}$ ) or neutronneutron $(r=\mathrm{n})$ interaction $^{\mathrm{a}}$ operator and third line represent proton-neutron interaction energy operator matrix element ( $s=\mathrm{n}$ if $r=\mathrm{p}$ and vice versa), the summations is over occupied $\left(0 s_{1 / 2}\right)$ one-particle states for given nucleon. Eq. (8) allows (1) to be expressed without $V^{(1)}=h^{(r)}$ matrix elements. There is also possibility to express (1) by (slightly transformed) $V^{(2)}$ elements only (see Supplementary Information). The connection between notations in (1-7) and (8-10) is

$$
\left|\psi_{i}^{(r)}\right\rangle=\hat{a}^{+}(\rho \cdot i)|0\rangle
$$

where $\rho=-1$ for $r=\mathrm{p}$ and $\rho=+1$ for $r=\mathrm{n}$, for the HF ground state wave-function

$$
|H F\rangle=\hat{a}^{+}\left(k_{Z}\right) \hat{a}^{+}\left(q_{A-Z}\right)|0\rangle,
$$

where for ${ }^{4} \mathrm{He}, k_{\mathrm{Z}}=k_{2}=(-1,-2), q_{\mathrm{A}-\mathrm{Z}}=q_{2}=(1,2)$ for the two lowest lying m-levels for each nucleon type.

For any realistic nucleon-nucleon potential there is no analytical solution for either HF eigen-problem or a general beyond-HF eigen-problem of the hamiltonian (1). The numerical solution is done by restriction to finite-dimensional one-particle subspaces (defined by index sets $\left.I^{(p)}, I^{(n)}\right)$. For most bounded state properties HF solution should serve as a mere $\mathrm{ON}$ one-particle basis set generating procedure and more realistic A-body wavefunctions (respecting correlations between particle motions) should be obtained through diagonalization of (1) within (subspace) of product space of restricted oneparticle state spaces for each particle. Due to the Ritz variational principle $[12,13]$, eigenvalues obtained are upper bounds to eigenvalues of (1) with respect to nonrestricted, complete space.

In case of (1) being diagonalized within all possible (appropriately anti-symmetrized) products of oneparticle functions (Configurations) term Full Configuration Interaction (FCI) is used $[11,14,15]$. In case only certain configurations are used, the method is called Limited Configuration Interaction and possible variants could be CIS, CISD, CISDT, ..., for set of at

\footnotetext{
a Please see Supplementary Information for the details. The two-body interaction contains also (second quantized form of) mass-polarization term $(1 / M) \mathbf{p}_{\mathrm{i}} \cdot \mathbf{p}_{\mathrm{j}}\left(\mathbf{p}_{\mathrm{i}}\right.$ being linear momentum of $i$-th particle) due to the
} CMS energy subtraction. most single, double, triple, ... excitations from ground state (or from set of model configurations in MRCI variant) [16]. FCI is size-consistent and therefore preferable over limited variants (even when calculated with respect to a greater one-particle sets), only FCI energy will converge with one-particle basis (limiting to completeness) to exact eigenvalue [16] of the original hamiltonian (1). The problem is, however, computational demands - since the number of configurations scales exponentially with nucleonic number, as $\mathrm{O}\left(N^{A}\right)$, where $N$ is size of one-particle basis set and $A$ number of particles.

There are three possible classes of solutions to aforementioned scaling problem - 1. To account for excited configurations approximately (e.g. via an exponential Ansatz for Wave-operator in Couple Clusters methods [17-20]), parametrize multiconfigurational wave-function in different way (e.g. as in Multi-configurational Self-Consistent Field MCSCF methods $[16,21]), 2$. To re-formulate bounded state problem within Density Functional Theory [22-24] or 3. Use a computational device itself based on rules of quantum mechanics [3-5,25-29] and therefore better suitable for eigen-value problem (1).

While the first two classes in fact avoid to calculate FCI energy itself, the only true solution is the last one. Proof-of-principle of a particular algorithm for a theoretical quantum computer device will be presented in the next section.

\subsection{Abrams-Lloyd Algorithm and Iterative Phase Estimation}

\subsubsection{Introduction}

While the first applications of Abrams-Lloyd algorithm [4] have been tailored for computational ( $a b$ initio) quantum chemistry [4-8] (e.g. FCI for electronic structure for fixed nuclei $[26,38]$, FCI for electrons and nuclei in molecule treated on equal footing via the NOMO approach [30]), the possibility to use AbramsLloyd algorithm for computational physics problem has been also previously suggested (e.g. [31]).

Here the main steps of the algorithm will be briefly reminded. The algorithm aim is to estimate hamiltonian $H$ eigenvalue $E$ for given multiparticle stationary state based on representation of evolution operator $U=\exp (-i$ $\Delta t H)$.

\subsubsection{Inputs for algorithm}

The inputs for algorithm consists of:

1. Bounds $E_{\min }, E_{\max }$ to estimated eigenvalue, such that $E_{\text {min }}<E<E_{\max }$ and desired accuracy in number of bits, $m$ (the usual choices would be $m=17$ to 27).

2. Estimate (initial guess) $\left|\Psi_{0}\right\rangle$ of corresponding eigenvector $|\Psi\rangle$ such that mutual overlap $S=\left|\left\langle\Psi_{0} \mid \Psi\right\rangle\right|^{2}$ is greater than $S_{\text {crit }}$ (in an initial formulation $S_{c r i t}=\pi^{2} / 16 \quad 0.62$ )

3. Elements of second quantized hamiltonian (1) 
The quantum algorithm is probabilistic and provides any of the two closest binary approximates $E_{b i n}$ or $E_{b i n}+2^{-}$ ${ }^{m}$, such that the correct result $E$ lies in between them $\left(E_{b i n}\right.$ $<E<E_{b i n}+2^{-m}$, or $E=E_{b i n}+2^{-m} \delta$, where $\left.\delta \in(0 ; 1)\right)$ with success probability $[3,29] p_{m}$

$$
p_{m} \geq S \cdot h_{m}(\delta),
$$

where overlap $S$ has been defined in second point of input list and factor $h$ depends on reminder $\delta$ and accuracy $m$ (bits),

$$
h_{m}(\delta)=\frac{1}{2^{2 m}}\left(\frac{\sin ^{2}(\pi \delta)}{\sin ^{2}\left(\pi \delta / 2^{m}\right)}+\frac{\sin ^{2}(\pi(1-\delta))}{\sin ^{2}\left(\pi(1-\delta) / 2^{m}\right)}\right),
$$

for any combination of $\delta$ and $m, h$ has a lower bound,

$$
h_{m}(\delta) \geq 8 / \pi^{2}
$$

therefore, the condition $S>S_{\text {crit }}$ is important to assure that $p_{m}>0.5$. By eventual repetition of quantum computer algorithm, the success probability can be then amplified as close to 1.00 as desired.

When the algorithm is just simulated for smaller number of one-particle basis states on a classical computer, the desired result and corresponding probability is well known and number of algorithm repetitions $r$ to achieve probabilities of, e.g., 0.99, 0.9999 or 0.999999 can be computed $^{\mathrm{c}}$ [30]. However, in case of real quantum computer, the basis size $N$ (e.g. cardinality of one-particle basis sets) might be huge, not allowing classical computer calculation in realistic time, so success probability might not be known prior to calculation itself. Therefore, it is important to do parametric study suggesting that chosen initial guess $\left|\Psi_{0}^{(N)}\right\rangle$ will not drop under $S_{\text {crit }}$ (e.g. based on extrapolation of overlap $S_{N}=\left|\left\langle\Psi_{0}^{(N)} \mid \Psi^{(N)}\right\rangle\right|^{2}$ from $N$ values for which classical simulation is possible to $N$ for which calculation will be done exclusively on quantum computer). Given extrapolated overlap $\mathrm{S}$ we can estimate $p_{m}$ and number of repetitions $r$ needed for result to be correctly decided from majority voting on (in each algorithm run) obtained binary expansions. In an unfortunate case, we would fail to choose correct eigenvector guess, the algorithm result is still not void, but provide us by the eigenvalue corresponding to eigenvector which has largest overlap with initial guess used.

\footnotetext{
${ }^{\mathrm{b}}$ Even though this expression is a lower bound to probability, in most cases, the true probability isn't much higher than bound. The possible difference is due to the "overlap" of probability distribution peaks associated to different eigenvalues of studied hamiltonian $H$. In a typical case, the gap between eigenvalue in question and its neighbors is by orders larger than read-out accuracy $\left(2^{-m}\right)$ and (13) can be considered as an equation.

${ }^{c}$ The upper bound to $r$ could be decided through binomial distribution of one-bit-based outcomes "correct eigenvalue"/" incorrect eigenvalue". Then according to Chernoff bound [32-34], should be

$$
r \leq d \cdot \frac{2 \ln (10) \cdot p_{m}}{\left(p_{m}-1 / 2\right)^{2}},
$$

where overall success probability from majority vote on all $r$ independent algorithm runs is $1-\varepsilon, \varepsilon=10^{-d}$ (a typical choice for $d=2,4$ or 6 [30]). Wider spread of $a$ register readouts allows for $r$ smaller than upper bound on right side of (16) [30].
}

Possible initial guess should have to be representable efficiently, i.e. through at most polynomially (in oneparticle basis set size) many unitary gates. The most straightforward choice would be Hartree-Fock (Mean Field) guess. However, for a multireference states (common for excited states but in case of small gap between highest occupied one-particle state and lowest unoccupied one or for open-shell systems possible for ground state as well) this choice might lead to $S<S_{\text {crit }}$ and more sophisticated initial guess would be recommended. First way would be beyond-Mean field eigenvector calculated with less computationally demanding method than FCI - e.g. CISD, CISDTQ, CCSD, MBPT or FCI $[11,16]$ with excitations restricted to given, limited number of unoccupied (virtual) spin orbitals (Fockian eigenvectors) within same one-particle basis set generated by Hartree-Fock calculation. Second, different one-particle basis set (but connected with the one used in quantum algorithm via finite-dimensional unitary transformation) might be generated from MCSCF or as Natural Orbitals (NO) through diagonalization of one-particle density matrix from Many Body Perturbation Theory (MBPT) of some higher order $[11,16]$. Third, purely quantum approach would be the use of Adiabatic State Preparation (ASP) method [35], [4], which facilitates transition from ground state (represented in quantum register) of one hamiltonian (here Hartree-Fock hamiltonian) to ground state (represented in quantum register) of another hamiltonian (e.g. FCI hamiltonian (1)) under preposition that smooth transformation of hamiltonian in question do exist and that ground state and lowest excited state will neither exchange nor degenerate inside the process (the lowest gap between them then limits number of steps needed).

\subsubsection{Algorithm description}

Abrams-Lloyd algorithm is based on the PhaseEstimation algorithm [3,29] for calculation of eigenvalues and eigenvectors of unitary matrix $U$, represented as a quantum logical gate acting on $l$ qubits of quantum register $b$ for storage of the eigenvector $|\psi\rangle$ (Fig. 1), this register is initialized to initial guess $\left|\Psi_{0}\right\rangle$ as mentioned in previous section. Quantum register $a$ is initialized to $|0\rangle|0\rangle \quad|0\rangle$ (m-times, for reference, please see the beginning of quantum wires on the left of Fig.1) state and serves to store bits $f_{1}, f_{2}, \ldots, f_{\mathrm{m}}$ of computed eigenvalue (please see the measurement symbols on the end (rightmost part) of wires in Fig. 1), then $E$ can be computed from linear equality

$$
\frac{E-E_{\min }}{E_{\max }-E_{\min }}=0 . f_{1} f_{2} \quad f_{m}+2^{-m} \delta,
$$

where $\delta \in(0 ; 1)$, or $2^{-m} \delta$ is a reminder limiting accuracy. An alternative using only one qubit read-out register $a$ exists and are discussed in [3,29-31,36-38] (Fig. 3 on page 3 [31]), as Iterative Phase Estimation $\mathrm{A}$ and $\mathrm{B}$ (IPEA A, IPEA B, [38]) - since $m$ qubit needed is traded for $m$-times repetition of ,decoupled” quantum circuit ([31], Fig. 3, page 3). 


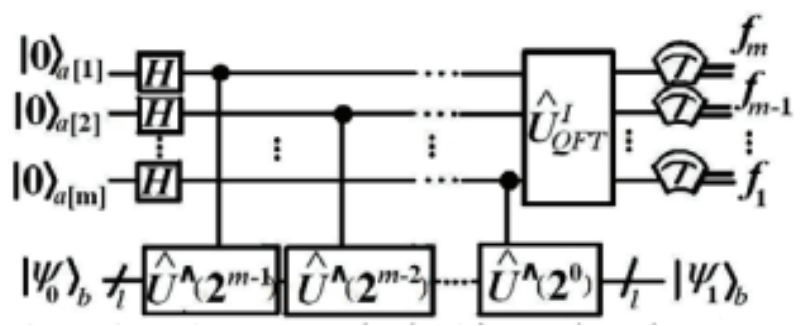

Fig. 1: Phase Estimation Algorithm (PEA) quantum logical circuit. Figure adopted from [31]

Inside the algorithm, the Hadamard gate,

$$
H=\frac{1}{\sqrt{2}}\left(\begin{array}{cc}
1 & 1 \\
1 & -1
\end{array}\right),
$$

is applied on each of $\mathrm{m}$ qubits of $a$ register (boxes with $H$ symbol in Fig. 1) creating equal weights superposition of binary numbers in $a$.

Then sequence of conditioned applications of quantum gate representation of $U$ on register $b$ is done (the lowest written wire in Fig. 1), with control qubit being from $a$ register (note the corresponding full black circles in the middle of Fig. 1). This creates entangled state $((2)$ in [31]) where all $x$-th powers of $U$ are entangled to corresponding binary representations of $x$ in $a$ register (19, first line).

The pre-last step of PEA is the inverse Quantum Fourier Transform (QFT, represented by a box with $\hat{U}_{Q F T}^{I}$ in Fig. 1, the best-known versions has computational time cost of $\mathrm{O}(m \log m)$ [39]) which in fact transform state vector in concatenated $a+b$ quantum register (denoted lcore $>$ as in [31]) from time domain (time is discretely sampled in the a register) to the energy domain,

$$
\begin{aligned}
\mid \text { core }\rangle & =\sum_{t} \exp \left(-i E_{j} t\right) c_{j}|t\rangle_{a}\left|\Psi_{(j)}\right\rangle_{b} \\
& \stackrel{Q F T^{\prime I}}{\longrightarrow} \sum_{E} c_{j}|E\rangle_{a}\left|\Psi_{(j)}\right\rangle_{b}
\end{aligned},
$$

where parametrization of unitary matrix $U$ in question as $U=\exp \left(-\mathrm{i} \Delta t\left(H-E_{\min }\right)\right)$ (with $\Delta t=2 \pi /\left(E_{\max }-E_{\min }\right)$ and $H$ being FCI hamiltonain matrix) is supposed from now, $E_{j}$ is exact FCI energy (minus $E_{\text {min }}$ ) for $(j+1)$-th state and $c_{j}$ is the coefficient of expansion of initial guess $\left|\Psi_{0}\right\rangle$ to exact FCI eigenvectors $\left|\Psi_{(j)}\right\rangle$, i.e.,

$$
S=\left|\left\langle\Psi_{0} \mid \Psi_{(0)}\right\rangle\right|^{2}=\left|c_{0}\right|^{2}
$$

The last step is a measurement of all $m$ qubits in $a$ register, denoted by "ح- $=$ =" in Fig. 1 - to obtain $m$ bits $f_{1}$, $f_{2}, \ldots, f_{m}$ binary representation of energy through (17). Since in previous step, the quantum core is in an entangled state, where, however, amplitude of component entangled to desired binary representation of a correct energy $E_{(0)}$ should dominate, this step will lead to correct result with probability $p_{m}$ given by (13).

At the end of the algorithm, the quantum register $a$ is in state corresponding to measurement outcome and eigenvector in question is encoded inside the quantum register $b$ as $\left|\psi_{1}\right\rangle_{b}$ (with the probability $p_{m}$ it is the eigenvector we searched for). As wave-function is not an observable, there is no simple way how to read it from quantum computer qubit-based memory. However, we could exploit it for some subsequent quantum algorithm - e.g. recover as much as possible information through Quantum Tomography [40-41].

All sources of quantum computer power superpositioning (Hadamard gate application), parallelism (ease to create all $2^{m}$ components in (19) in $m$ steps), entanglement (the entangled state in (19)) and destructive interference (the inverse QFT) are exploited in PEA and this would lead to an exponential speed up for FCI energy calculation when compared to classical computer-based procedure... under an important preposition that both initial eigenvector guess and evolution operator $U$ application (in general it is a $l$-qubit gate and should be decomposed into elementary one and two-qubit gates from universal quantum logic gate set [3]) will be represented efficiently (i.e. in number of time-steps (elementary gates) polynomial in one-particle basis set cardinality $N$ ). The latter preposition and $U$ gate decomposition will be discussed in following section 2.2.4 and the initial sate preparation in 2.2.5

\subsubsection{Computational complexity}

The $U$, exponential of shifted and scaled hamiltonian (1), can be rewritten in a simplified form as

$$
\hat{U}=\exp \left(i \Delta t \sum_{X=1}^{\Lambda} \hat{h}_{X}\right)=\exp \left(i \frac{\Delta t}{q} \sum_{X=1}^{\Lambda} \hat{h}_{X}\right)^{q}
$$

where $h_{X}$ are hermitian combinations of terms from expansion (1), e.g. for some $X=X_{0}$,

$\hat{h}_{X_{0}}=\hat{a}^{+}(i) \hat{a}^{+}(j) \hat{a}(k) \hat{a}(l)+\hat{a}^{+}(l) \hat{a}^{+}(k) \hat{a}(j) \hat{a}(i)$

Furthermore, in accordance to the Trotter formula $[3,37,42,43]$ for second order

$$
\begin{aligned}
& \exp (i(A+B) \Delta t)-o\left(\Delta t^{3}\right)= \\
& =\exp (i A \Delta t) \exp (i B \Delta t) \exp \left(\frac{1}{2}[\hat{A}, \hat{B}] \Delta t^{2}\right)
\end{aligned}
$$

the expansion should be partitioned into largest commuting hermitian blocks to minimize number of terms for the next step. In this step, Trotter-Suzuki formula $[3,37,42,43]$ is applied, with general $\eta$-th order form

$$
\begin{aligned}
& \exp \left(i \frac{\Delta t}{q} \sum_{X} \hat{h}_{X}\right)= \\
& =\prod_{k=1}^{2 \Lambda \eta} \exp \left(i \frac{\Delta t}{q} \theta_{k, p(k)} \hat{h}_{p(k)}\right)+o\left(\left(\frac{\Delta t}{q}\right)^{\eta}\right)
\end{aligned}
$$

where $\theta_{k, p(k)}$ are real coefficients fulfilling $\sum_{k: p(k)=p} \theta_{k, p(k)}=1, \Lambda$ is number of terms in operator sum in (21) and $p$ is projection from set $\{1,2, \ldots, 2 \Lambda \eta\}$ on set $\{1,2, \ldots, \Lambda\}$. For $\theta_{k, p(k)}$ value determination, please see [44],[3]. As discussed in [37], the order $\eta$ should be 
optimized. Too low order will provide an inaccurate approximation to the evolution operator $U$, yet too high order will lead to greater accumulated error (in classical computer simulation arising from rounding, in quantum computation from gate application error and decoherence). The optimization can be formulated (for an ideal quantum computer) as a minimization of $\eta q$ product (since the total number of exponential terms used to represent unitary operator $U$ by elementary quantum gates is equal to $\Lambda \eta q$ and $\Lambda$ is a fixed constant, for minimization technical details, see formula (296) in [37] and Fig. 7 there).

Then, each creation and annihilation operator in (22) or in any $h_{X}$ term is represented via quantum gates acting on register a storing the eigenvector of $U$.

In case of the direct mapping the $b$ quantum register qubits simply store the occupation numbers of m-levels (one-particle state characterized in Harmonic Oscillator scheme by fixed $n, l, j$ and $m$ quantum numbers) and since nucleons are fermions the binary character of qubits is well fit for this representation (occupation numbers are in $\{0 ; 1\})$. Let us denote the total number of m-levels used in suggested calculation as $N_{m}$. As an example, wave-function representation for ${ }^{4} \mathrm{He}$ nuclei is

$$
\begin{aligned}
& \left|\psi\left({ }^{4} \mathrm{He}\right)\right\rangle=c_{H F}|1\rangle|1\rangle|0\rangle|0\rangle \quad|1\rangle|1\rangle|0\rangle|0\rangle+ \\
& \mathrm{N}_{\mathrm{m}} \text { qubits for protons } \mathrm{N}_{\mathrm{m}} \text { qubits for neutrons } \\
& +\sum_{i} c_{i}\left|n_{0}^{p}\right\rangle\left|n_{1}^{p}\right\rangle \quad\left|n_{N_{m}}^{p}\right\rangle\left|n_{0}^{n}\right\rangle\left|n_{1}^{n}\right\rangle \quad\left|n_{N_{m}}^{n}\right\rangle
\end{aligned}
$$

where $n_{j}^{p}$ is occupation number for $j$-th m-level for protons, $n_{j}^{n}$ for neutrons, the sum runs over $2^{\wedge}\left(2 N_{m}\right)-1$ components, but only $\left(\begin{array}{c}N_{m} \\ 4\end{array}\right)^{2}$ of them has non-zero $c_{i}$, or are further relevant (since we suppose no-sea approximation, $\left.\sum_{\mathrm{j}} n_{j}^{p}=\Sigma_{\mathrm{j}} n_{j}^{n}=2\right)$. The size of $b$ quantum registers $l=$ $2 N_{m}$.

In the direct mapping case, creation and annihilation operators are represented by strings of Pauli matrices (Jordan-Wigner operator algebra mapping [45] employs $\mathrm{O}\left(N_{m}\right)$ gates, while more economic Bravyi-Kitaev mapping [46] needs just $\mathrm{O}\left(\log N_{m}\right)$, please see section 2.5.2.1 in [31]) acting on individual qubits of $b$ register.

While the direct mapping is qubit cost uneconomic (there are exponentially many more unphysical components in (25) than actively used in quantum computation!), the gate implementation is very straightforward and computational time is at most polynomial in $N_{m}$ (if initial state preparation is efficient).

Computational time can be estimated by

$$
t_{Q} \approx 2^{m} \cdot T e \cdot \frac{\Lambda}{N_{p}} \cdot N_{1},
$$

where $2^{m}$ arise from PEA circuit (Fig. 1), $T e=\eta q$, stands for number of terms in Trotterization of $U=\exp (-\mathrm{i} \Delta t$ $H), \Lambda$ stands for number of terms in second quantized hamiltonian (1), (21), $N_{p}$ for the number of them to be executable in parallel and $N_{l}$ for the number of elementary gates needed to represent exponential of one single term $h_{X}$ in (21). For general case of at most $k$-body forces in hamiltonian (1), $\Lambda=O\left(N_{m}^{2 k}\right)$, since gates with non-overlapping target qubits could be applied in parallel, for Bravyi-Kitaev [46,31] mapping $N_{l}=\mathrm{O}\left(\log N_{m}\right), N=\mathrm{O}\left(N_{m} / \log N_{m}\right)$ and for $\eta q=O\left(N_{m}^{\varepsilon}\right)$, where the power $\varepsilon$ is shown to be at worst $\varepsilon=6$ for two-body forces hamiltonian [47], but realistic estimates on molecular system provides $\varepsilon \in\langle 1.5 ; 2.5\rangle$ [48] the estimate (26) become

$$
t_{Q} \approx O\left(\left(\log N_{m}\right)^{2} \cdot N_{m} \wedge(2 k-1+\varepsilon)\right),
$$

when compared to classical diagonalization time for FCI hamiltonian matrix which is

$$
t_{C} \approx O\left(N_{m} \wedge(3 A)\right),
$$

considering the diagonalization has general scaling as $\mathrm{O}\left(n^{3}\right)$ for $n \times n$ matrix [49] (through for sparse matrices complexity decrease) and suggesting m-level scheme to be employed for classical computation as well (through that is ineffective) with upper bound to state number to be $\mathrm{O}\left(N_{m} \wedge A\right)$ with nucleon number $A$. When slowly rising logarithmic term is omitted, powers in (27) and (28) can be compared and for

$$
A \geq \frac{2 k-1+\varepsilon}{3} \in(1.5 ; 4),
$$

the quantum algorithm should be quicker than the classical one. However, since classical FCI diagonalization is done in $\mathrm{j}$-scheme and FCI Hamiltonian sparsity is exploited, the denominator in (29) should decrease to 2 or 1.5 , leading to critical $A$ in interval (3; 8 ), where upper bounds are estimated with $k=3$ and $\varepsilon=$ 7. But it can be concluded that quantum FCI will be beneficial, if not from ${ }^{4} \mathrm{He}$, from ${ }^{16} \mathrm{O}$ with certainty.

\section{Numerical tests}

In so far only classical FCI and limited CI preliminary calculations has been done for ${ }^{4} \mathrm{He}$, In our calculations we use the nucleon-nucleon optimized chiral potential NNLO_opt. The coupling constants of this interaction were determined by a new optimization method in order to minimize the effects of the three-nucleon force [50], the results are summarized in the following table,

Table 1. Selected ${ }^{4} \mathrm{He}$ ground state $\left(0^{+}\right) \mathrm{CI}$ energies $\left(E_{C I}\right)$ for NNLO_opt potential with $\Omega=16 \mathrm{MeV}$ HO basis set parameter, Hartree-Fock $\left(E_{H F}\right)$ and second order Many Body Perturbation Theory $\left(E_{M B P T}\right), N$ denote maximal major shell number ${ }^{\mathrm{e}}$ and $\left|c_{0}\right|^{2}=S$ the overlap between CI wave-function and Hartree-Fock ground state

\begin{tabular}{|c|c|c|c|c|c|}
\hline$N$ & $E_{H F}[\mathrm{MeV}]$ & $E_{M B P T}[\mathrm{MeV}]$ & $C l$ type & $E_{C I}[\mathrm{MeV}]$ & $\left|c_{O}\right|^{2}$ \\
\hline 1 & $-11,139290$ & $-12,945510$ & & & \\
\hline
\end{tabular}

\footnotetext{
${ }^{\mathrm{d}}$ Note that $N_{m}$ might not be considered as independent to $A$. At least $N_{m}$ $>A / 2$ for a non-zero number of virtual one-particle states in FCI calculation. However, the $N_{m}$ for fixed error in energy might not need to grow more than linearly in $A$, that is $N_{m}=\alpha A+\beta$ for some small positive $\alpha$ and $\beta$. Number of m-shells, $N_{m}$, is in relation to number of major shells, $n$, as $N_{m} \sim \mathrm{O}\left(n^{3}\right)$, but number of j-shells as $N_{j} \sim \mathrm{O}\left(n^{2}\right)$, therefore $N_{j} \sim \mathrm{O}\left(N_{m}^{2 / 3}\right)$, further explaining change of denominator in (29).

${ }^{\mathrm{e}} N=2 n+l$, where $n$ starts from zero, e.g. $N=2$ means collection of $\mathrm{j}$ shells $0 \mathrm{~s}_{1 / 2}, 0 \mathrm{p}_{3 / 2}, 0 \mathrm{p}_{1 / 2}, 1 \mathrm{~s}_{1 / 2}, 0 \mathrm{~d}_{5 / 2}$ and $0 \mathrm{~d}_{3 / 2}$.
} 


\begin{tabular}{|c|c|c|c|c|c|}
\hline 2 & $-13,542445$ & $-16,280365$ & $\mathrm{FCl}$ & $-17,590998$ & 0,726 \\
\hline 3 & $-13,542445$ & $-19,754664$ & $\mathrm{CISD}$ & $-20,116542$ & 0,853 \\
\hline 5 & $-15,496509$ & $-24,637898$ & & & \\
\hline 8 & $-15,834727$ & $-26,024630$ & $\mathrm{FCI}(2)$ & $-16,188135$ & 0,987 \\
\hline 8 & & & $\mathrm{CISD}(3)$ & $-16,867806$ & 0,971 \\
\hline & Exp. [51] & $-28,296$ & & & \\
\hline
\end{tabular}

where round braces indicate major shell cut-off subsequent to Hartree-Fock basis generation. HartreeFock virtual one-particle states are in general rather problematic basis for correlation energy convergence as comparison of FCI for $N=2$ with FCI(2) for $N=8$ shows

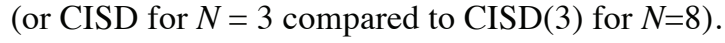

However, the overlap $S=\left|c_{0}\right|^{2}=\left|\left\langle H F \mid C I, 0^{+}\right\rangle\right|^{2}$ and resulting PEA (quantum eigenvalue estimation) success probability estimates (Tab. 2) increase (e.g. from FCI to FCI(2)) as one-particle basis set (of a fixed cardinality) is generated through HF closer to HF limit.

Table 2. Phase Estimation Algorithm success probabilities for CI calculations from Tab. 1

\begin{tabular}{|l|c|c|c|c|l|}
\hline$N$ & $\mathrm{Cl}$ type & $p_{\min }$ & $p$ & $p_{\max }$ & $\left|c_{1}\right|^{2} /\left|c_{0}\right|^{2}$ \\
\hline 2 & $\mathrm{FCl}$ & 0.588 & 0.702 & 0.726 & $1.52 \cdot 10^{-3}$ \\
\hline 3 & $\mathrm{ClSD}$ & 0.691 & 0.828 & 0.853 & $6.52 \cdot 10^{-3}$ \\
\hline 8 & $\mathrm{FCl}(2)$ & 0.800 & 0.804 & 0.987 & $1.02 \cdot 10^{-3}$ \\
\hline 8 & $\mathrm{CISD}(3)$ & 0.787 & 0.845 & 0.971 & $1.32 \cdot 10^{-3}$ \\
\hline
\end{tabular}

As an example, $\left|c_{1}\right|^{2} /\left|c_{0}\right|^{2}$, relative configuration weight for the second most abundant configuration (for the first two rows in Tab. 2 corresponding to $0 \mathrm{~s}_{1 / 2} \rightarrow 1 \mathrm{~s}_{1 / 2}$ proton monoexcitation (for neutrons with by only $0.7 \%$ higher $\left.\left|c_{j}\right|\right)$, for the last two corresponding to biexcited configuration (one proton and one neutron from $0 \mathrm{~s}_{1 / 2}$ do $\left.0 p_{3 / 2}\right)$ is presented in Tab. 2 as well.

Bounds to probabilities, $p_{\max }=S=\left|c_{0}\right|^{2}, p_{\min }=S /\left(2 S_{c r i t}\right)$ and $p$ are estimated from (13) with read-out parameter $m=20$ and $\delta$ derived from $E_{m a x}=E_{H F}$ and $E_{m i n} \approx E_{H F}+\alpha$ $\left(E_{M B P T}-E_{H F}\right)$ (with $\alpha=2$ ). For a more detailed description of wave-function expansion into configurations for both ground and few chosen excited states, please see the Supplementary Information.

\section{Conclusion}

Preliminary classical CI calculations indicate quantum Abrams-Lloyd algorithm feasible for ${ }^{4} \mathrm{He}$ ground state FCI calculations, (at least for smaller basis set size) with the simplest single-determinantal initial eigenvector guess (identical to Hartree-Fock solution). For excited states, linear combination of a smaller set of configuration should be used as an initial eigenvector guess. As will be listed in following section, the feasibility studies should carry on for ${ }^{4} \mathrm{He}$ and later to be extended to larger nuclei. Different quantum algorithms than Abrams-Lloyd should be investigated as well.

\section{Future prospects}

1. To extend calculations to a greater basis set,

2. perform a full simulation classical of Abrams-Lloyd algorithm,
3. to provide reliable and efficiently computable lower bounds $E_{\text {min }}$ (e.g. based on Tempel [52], Weinstein or Stevenson formulae [53] employing $<\mathrm{H}^{2}>$ or on Inner Projection technique [54]).

We would like to thank Dr. Libor Veis from J. Heyrovský Institute of Physical Chemistry in Prague and MSc. Michaela Chovancová from Department of Physics and Technology, University of Bergen for technical support and consultations, TESNAT 2017 Organizing Committee for hospitality and the opportunity to present this work. Access to computing and storage facilities: National Grid Infrastructure MetaCentrum (www.metacentrum.cz/en/index.html) under program CESNET LM2015042 is greatly appreciated.

\section{References}

1. B. Barrett, et al, Prog Part Nucl Phys 69 (2013) 131

2. https://indico.cern.ch/event/609505/contributions/2517689/attach ments/1448184/2231965/Mala_Skala_2017_Knapp.pdf

3. M.A. Nielsen, I.L. Chuang, Quantum Computation and Quantum Information (Cambridge University Press), (2000).

4. D.S. Abrams, S. Lloyd, Phys.Rev.Lett. 83, 5162 (1999).

5. A. Aspuru-Guzik, et al, Science 309, 1704 (2005).

6. H. Wang, et al, Phys. Chem. Chem. Phys. 10, 5388-5393 (2008)

7. J.D. Whitfield, et al, Mol. Phys. 109, 735 (2011).

8. L. Veis, J. Pittner, J. Chem. Phys. 133, 194106 (2010).

9. P.-O. Löwdin, Phys. Rev. 97, 1474 (1955).

10. D.J. Thouless, Nucl. Phys. 21, 225 (1960).

11. J. Suhonen, From Nucleons to Nucleus: Concepts of Microscopic Nuclear Theory, Springer, Berlin, (2007).

12. W. Ritz, J. Reine, Angew. Math., 135 (1908) 1-61.

13. https://quantummechanics.ucsd.edu/ph130a

14. Q. Chong, J Phys Conf Ser 742 (2016) 012030.

15. Sternberg et al, Accelerating Full Configuration Interaction Calculations for Nuclear Structure in SC '08: Proc. of the 2008 ACM/IEEE Conf. on Supercomputing. https://www.osti.gov.

16. A. Szabo, N. S. Ostlund, Modern Quantum Chemistry: Introduction to Advanced Electronic Structure Theory, McGrawHill Publishing Company, New York, (1989).

17. G. Hagen et al, Rep. Prog. Phys. 77, 096302 (2014).

18. O. Sinanoğlu (1962) J Chem Phys 36 (3) 706.

19. J. Čížek (1966) J Chem Phys 45 (11): 4256.

20. I. Shavitt, R.J. Bartlett (2009) Many-Body Methods in Chemistry and Physics: MBPT and Coupled-Cluster Theory. Cambridge University Press. ISBN 978-0-521-81832-2.

21. J. Lietz, etal., "Computational Nuclear Physics and Post HartreeFock Methods,", Lecture Notes in Physics (Springer), "An advanced course in computational nuclear physics: Bridging the scales from quarks to neutron stars", M. Hjorth-Jensen, M. P. Lombardo, U. van Kolck, Editors, (2016) [arXiv:1611.06765].

22. P. Hohenberg, W. Kohn, Phys. Rev. 136 (3B): B864-B871, (1964).

23. W. Kohn, L. J. Sham, Phys. Rev. 140 (4A): A1133-A1138 (1965).

24. J. Dobaczewski, J Phys Conf Ser 312:092002(2011).

25. R. P. Feynmann, Int J Theor Phys, 21, 6/7, (1982).

26. B. P. Lanyon et al, Nat Chem 2, 106-111 (2010).

27. D. S. Abrams, S. Lloyd. Phys. Rev. Lett., 81:3992-3995, (1998). quant-ph/9801041.

28. J. Gruska: Quantum Computing, Advanced Topics in Computer Science Series, The McGraw-Hill Companies, ISBN 007709503 0.F (1999).

29. M. Dobšíček: Quantum computing, phase estimation and applications, Ph.D. thesis, Czech Technical University in Prague, Faculty of Electrical Engineering, Department of Computer Science and Engineering, (2008).

30. L. Veis et al Int J Quantum Chem (2016), 116, 1328-1336. DOI: 10.1002/qua.25176.

31. J. Višňák, EPJ Conferences 100, 01008 (2015).

32. H. Chernoff, (1952) Ann Math Stat 23 (4): 493-507.

33. T. Hagerup, C. Rüb, (1990) Inform Process Lett 33 (6): 305.

34. F. Nielsen, (2011), arXiv:1102.2684

35. L. Veis, J. Pittner, J Chem Phys, 140, 214111 (2014).

36. A. Yu. Kitaev (1995). arXiv:quant-ph/9511026. 
37. J. Višňák, Quantum chemical algorithms for Quantum Computers, Diploma Thesis, Mathematical-Physical Faculty, Charles University in Prague (In Czech, 2012).

38. L. Veis et al, Phys. Rev. A 85, 030304(R) (2012).

39. L. Hales, S. Hallgren, Proc. of the $41^{\text {st }}$ An. Sympos. on Foundations of Comp. Sci., p. 515, (2000),

40. R. Blume-Kohout. arxiv.quant-ph/0611080 (2006).

41. J. Řeháček, Z. Hradil, M. Ježek, M. (2001). Phys. Rev. A. 63: 040303.

42. H. F. Trotter, Proc Am Math Soc 10:545-551, (1959).

43. P. R. Chernoff, J Funct Anal, 2:238-242, (1968).

44. Dobšíček M et al, Quantum Simulat ions: Report, Technical report, Microtechnology and Nanoscience, MC2, Chalmers, S41296 Göteborg, Sweden, (2010).

45. P. Jordan, E. Wigner, Z. Phys., 47, Issue 9-10, pp 631-651 (1928).

46. S.B. Bravyi, A.Y. Kitaev, Ann. Phys. 298, Iss. 1, 210-226(2002).

47. D. Wecker, B. Bauer, B.K. Clark, M.B. Hastings, M. Troyer, Phys. Rev. A 90, 022305 (2014).

48. D. Poulin, et al, arXiv:1406.4920v1 (2014).

49. W.H. Press, S.A. Teukolsky, W.T. Vetterling, B.P. Flannery, (1992). Numerical Recipes in C (2nd ed.). Cambridge University Press. ISBN 0-521-43108-5.

50. A. Ekstroem et al (2013) Phys Rev Lett 110192502.

51. G. Audi et al (2017) Chinese Phys. C 41030001.

52. L. M. Delves (1972) J. Phys. A: Gen. Phys. 51123.

53. Löwdin P.-O. and Quantum Chemistry Group (1965): Studies in Perturbation Theory. X. Lower Bounds to Energy Eigenvalues in Perturbation-Theory Ground State. Uppsala University, Sweden

54. F. Vinette, J. Čížek (1991) J Math Phys 32, 3392. 